\title{
Recovery Time and Propagation Effects of Passenger Transport Disruptions
}

\author{
Caterina Malandri ${ }^{\mathrm{a}^{*}}$, Achille Fonzone ${ }^{\mathrm{b}}$, Oded Cats ${ }^{\mathrm{c}}$ \\ ${ }^{a}$ University of Bologna, Viale Risorgimento 2, 40136, Bologna, Italy \\ ${ }^{b}$ Edinburgh Napier University, 10 Colinton Road, EH10 5DT, Edinburgh, UK \\ ${ }^{c}$ Delft University of Technology, The Netherlands
}

\begin{abstract}
We propose a method to evaluate public transport network vulnerability. We study the evolution of the passenger Volume Over Capacity (VOC) ratio throughout the network to measure the spatial and temporal extent of the impacts caused by an unplanned service segment disruption. The VOC ratio provides an indication of the on-board travel comfort, an important level-of-service indicator, as well as reflects the residual capacity for absorbing additional demand. Because of the dynamic nature of public transport systems, disturbances propagate through the network in both time and space. Our modeling approach is able to capture transit system dynamics and quantify the extent to which the network exhibits spillover effects. We apply the method to the case of the rapid public transport system of Stockholm, Sweden. We demonstrate how the changes in network saturation and the corresponding recovery time can be quantified.
\end{abstract}

Keywords: Vulnerability; Public transport; Recovery; Spillover; Flow propagation. 


\section{Introduction}

Society relies on the supply of a number of critical infrastructure systems - such as electric power, water supply, communication and information networks - which have become gradually more complex and interdependent. If the performance of one of those systems is reduced considerably, society can suffer extremely severe consequences. The transport system is critical for societies in developing and developed countries as it enables the movement of people and goods between origins and destinations.

The research field related with the risk of severe transport network disruptions and their impacts is generally called vulnerability analysis. The term "vulnerability" refers to the susceptibility of a system to experience severe performance impacts in consequence of exceptional disruptions, unlike "unreliability" which refers to inherent and recurrent performance variations. "Robustness" is the antonym of vulnerability and therefore describes the capability of a system to absorb shocks and withstand disruptions. Taleb (2014) suggested that systems should aspire to become anti-fragile by getting more robust, i.e. more able to cope with shocks, rather than aim for a rigid design that prevents shocks from occurring altogether. While both vulnerability and robustness refer to the consequences of disruptions on network functionality once they occur, "resilience" demands also a rapid recovery back to normal operations and performance (Barker et al. 2013). Resilience analysis must thus consider the deterioration of system functionality and performance caused by an initial negative event, "incident", which may provoke consequences beyond the specific location and time at which the incident occurs. For example, on January 18, 2018, gusts of up to $140 \mathrm{~km} /$ hour have caused the cancellation of the majority of trains in the Netherlands, more than 6 hours after the adverse weather conditions were encountered. Hence, the notion of network resilience calls for the analysis of system dynamics and the development of advanced methods for representing supply and demand time-dependency and analysing network flow (re-)distribution in the event of a disruption.

This study focuses on public transport networks, which is a fundamental component of transport systems. Because of the growth in population, travel demand and motorization, traffic induces growing externalities such as increasing congestion and negative environmental impacts. Public transport may alleviate some of the negative consequences of mobility. However, to be an attractive alternative for travelers, it must be able to withstand or quickly recover from disturbances such as infrastructural and vehicular malfunctions. Knowledge about the distribution and magnitude of the impacts of potential service disruptions is crucial to effectively allocate resources for the prevention, mitigation and restoration of disruption of public transport services. A large variety of incidents may afflict public transport systems: they could be unexpected, provoked voluntarily or involuntarily by men or triggered by natural phenomena. They may span from adverse weather to terror attacks, technical failures, strikes or the breakdown of physical components.

Public transport network vulnerability has been often studied in terms of network topology. In these studies, the public transport system is represented as a graph where nodes, corresponding to stations or stops, are connected by links which represent service segments (von Feber et al., 2009; Berche et al., 2009). Disruptions are then simulated by removing graph's elements (nodes or links) randomly or by means of “directed attacks", i.e. selecting and deactivating links or nodes according to different centrality measures, such as the highest degree or betweenness centrality (Holme et al., 2002). After each removal, vulnerability is evaluated in terms of the decrease in network's performance, measured as the change of some selected topological and connectivity properties. For example, Latora and Marchiori (2005) evaluate the vulnerability of the Boston subway transportation system as the change of the network efficiency, i.e. the average of the reciprocal of the shortest distances between all node pairs in the network. As an alternative performance indicator, the relative size $S$ of the largest connected component of the network is used by Angeloudis and Fisk (2006) and Han and Liu (2009) to assess the vulnerability of twenty of the world largest subway networks and of ten Chinese subway systems, respectively. In a similar work, von Feber et al. (2012) compare the public transport systems of London and Paris in terms of vulnerability, introducing as indicator the area under the curve $S(c)$ as a function of the fraction $c$ of removed nodes or links. The same approach is applied by Zhang et al. (2011) in their study of the topological vulnerability of the Shanghai subway network. Derrible and Kennedy (2010) analyse the robustness of 33 metro systems worldwide in terms of the number of cyclic paths available in the network, an indicator of network redundancy.

The analysis of public transport network vulnerability using a strictly topological approach has considerable shortcomings. Such studies neglect a large number of factors, most importantly the notion of lines and their implications for passenger route choice, line operations and the need to transfer. Topological studies effectively assume that the removal of a link is equivalent to the network without this link to start with, with the remaining segments supposed to continue functioning independently. However, unplanned disruptions can cause adverse effects because service providers and users can not adjust to them upfront. Travel demand and in particular the effect on passenger rerouting and the number of affected users need to be explicitly considered. The availability of information about alternative routes and the duration 
of the incident is expected to play an important role in determining the consequences of public transport disruptions. Moreover, the dynamics of public transport system lead to the propagation of disturbances across the network due to knock-down effects on infrastructure and rolling stock and spillover effects due to the redistribution of passengers flows and capacity limitations. It is thus crucial to represent the interaction between supply and demand and their inherently stochastic processes in the public transport system.

To overcome these drawbacks, recently some vulnerability studies which model network loading have been developed, allowing the evaluation of additional important factors such as the level of congestion and delays. For example, Rodriguez-Nunez and Garcia-Palomares (2014) evaluate consequences of disruptions as the increase in average travel time, assuming that diverted travellers choose the fastest route available (i.e. all-or-nothing assignment). In addition, impacts of disruptions are analysed in terms of missed trips, i.e. the number of trips which cannot be completed because of the disturbance. Similarly, De-Los-Santos et al. (2012) estimate the increase of the overall travel time of all passengers in the network after a link failure. Cats and Jenelius (2015) developed a detailed dynamic robustness analysis using a dynamic agent-based transit assignment model. The latter is used to assess the increase in overall changes in passenger welfare measured in terms of total generalized travel costs. In Taleb (2014) systems are considered vulnerable if the negative impacts increase disproportionally to the magnitude of capacity reductions. The existence of this kind of vulnerable systems is shown by Cats and Jenelius (2016), where unplanned service disruptions with half the capacity reduction resulted in more than half as much delay. Shelat and Cats (2017) propose two local link criticality indicators for measuring spillover effects and analyse them for planned disruptions using a static stochastic user equilibrium transit assignment model.

In this study, measures of resilience in terms of the extent to which disruption impacts propagate across the network and time to recovery are defined and applied for the analysis of disruptions in a metropolitan public transport system. We model the problem in a dynamic framework with a time-dependent network representation. Our flow distribution model includes a behavioural route choice model mimicking passengers en-route travel decisions. We study the change in link saturation measured in terms of the passenger volume over capacity ratio throughout the network after a disruption. Disruptions causing comparable overall impacts in terms of total time loss may have different effects on network saturation and hence capacity to absorb disruptions depending on the operational state prior to the disruption on each network element. Impacts on networks performance are evaluated in terms of magnitude and duration, and the recovery time - the time that elapses before the system rebounds to its initial performance - is estimated.

The key contributions of this study are: (1) developing a framework for assessing the temporal and spatial propagation of service disruptions in a public transport network; (2) illustrating the method by an application to the Stockholm network. In the application, we analyse a range of disruption scenarios, determining time for recovery based on the trends of flow re-distribution and quantifying the geographical extent of spill-over effects based on flow propagations. The results of the application show that the impacts of disruptions are not confined to the immediate aftermath of the disruption and the area near the source of the disruption. The investigation of the recovery time and the geographical scale affected by various disruptions can support tactical and operational decisions concerning preparation and mitigation measures. Such measures include determining the availability of switches that allow for short-turning, timetable design, the allocation of reserve vehicles and the degree of isolation in infrastructure and operation between different sub-network components.

In the following, the model is briefly presented followed by the vulnerability indicators proposed in this study for analysing the extent of disruption spill-over effects (Section 2). The results are then presented for a case study in Stockholm, Sweden (Section 3), followed by concluding remarks (Section 4).

\section{Methodology}

\subsection{Modelling public transport dynamics}

Transit systems consists of various components that interact through numerous processes. The model used in this study considers the interaction between traffic dynamics, transit operations and traveller decisions.

The transport network lies on top of the layer of the relevant physical network of roads and railways. It is represented by a direct graph $G(S, E)$ where $S$ is the set of nodes, corresponding to stops, and $E$ is the set of links, representing connections between stops. The walking distances between stops are given to allow stop choice and transfers, without the explicit representation of pedestrian network links. Each link $e$ may be operated by one or several public transport lines. A transit line $l$ is defined by a sequence of stops or stations $l=\left(s_{l 1}, s_{l 2}, \ldots, s_{l|l|}\right)$, where $s_{l 1}=o_{l}$ is the origin stop and $s_{l|l|}=d_{l}$ is the destination stop. Similar to vehicle lines, the physical path of a traveller is defined by a sequence of stops 
from the origin to the destination, that is $j=\left(s_{j 1}, s_{j 2}, \ldots, s_{j|j|}\right)$, where the origin stop is $o_{j}=s_{j 1}$ and the destination stop is $d_{j}=s_{j|j|}$.

The service supply is modelled in terms of vehicle runs. Each line has a timetable and vehicles follow a schedule that consists of a sequence of trips. Because of the dependency between successive trips, delays may propagate from one trip to other. Vehicle trip travel times consist of two parts: riding times on links and dwell times at stops. Riding times are composed of running times on links and delays at intersections. In this study, the effects of the background traffic are modelled implicitly by representing link travel times as random variables with distributions that are derived from empirical travel times of public transport vehicles. Delays at intersections are determined by individual stochastic queue servers, that generate service times following empirically estimated distributions. Dwell time is the time a vehicle spends at a stop to let passengers board and alight. It depends on the number of passengers boarding and alighting, on the type of stop (bay/in-lane) and on the vehicle type. In addition, the payment method and the boarding regime are important determinant of the dwell time. Supply and demand interact dynamically. The effects of demand on supply are manifested mainly through the dwell time. In addition, passenger path choices are influenced by how the public transport system evolves.

Passenger demand is represented by an OD matrix at stop level. Trips are initiated at an origin stop and passengers have to choose their path to a pre-defined destination stop. Each passenger when travelling undertakes successive path choice decisions that depend on the evolving public transport system conditions. The evaluation of alternative paths depends on traveller's preferences and expectation, determined by prior knowledge, experience and the availability of real-time information. It is assumed that, in the context of high frequency urban public transport systems, travellers have a prior knowledge of network topology, timetable travel times and planned headways. Travellers' ability to carry out a decision is also subject to vehicle capacity constraints. All travellers' decisions are represented with a multinomial Logit model. The deterministic part of the utility function of passenger $n$ associated with path $i$ is denoted $v_{i n}$ :

$$
v_{\text {in }}=\beta_{i}^{\text {wait }} t_{\text {in }}^{\text {wait }}(t)+\beta_{i}^{\text {ivt }} t_{\text {in }}^{\text {ivt }}(t)+\beta_{i}^{\text {walk }} t_{\text {in }}^{\text {walk }}+\beta_{i}^{\text {trans }} \text { trans }_{i}
$$

Where $t_{i n}^{\text {wait }}(t)$ and $t_{i n}^{\text {ivt }}(t)$ are the expected waiting time and in-vehicle time; $t_{i n}^{\text {walk }}$ is the expected walking time; trans $_{i}$ is the number of transfers in the path. $\beta_{i}$ are the corresponding weights. The dynamic path choice model includes three decision models: connection, boarding and alighting. A connection decision takes place when the traveller chooses the start and/or alighting point of their trip. In both cases, the traveller choses between alternative stops by evaluating path alternatives which connect each candidate stop with the traveller's final destination stop. The traveller can choose between staying at the same stop and waiting for another transit service, walking to a nearby stop in order to wait there for another transit service or walk directly to the final destination. A boarding decision is made when the traveller waits at a stop and a vehicle arrives. The traveller can choose whether to board it or stay at the stop and wait for another vehicle. Once onboard a vehicle, a traveller makes an alighting decision which may be reconsidered in light of new information.

For more information on the dynamic route choice model and the consideration of congestion effects, the reader is referred to Cats et al. (2016).

\subsection{Implementation}

The supply and demand model is implemented using a mesoscopic pubic transport simulator called BusMezzo. The agentbased model is able to represent the interactions between traffic, public transport operations and traveller decisions. The latter are represented as a sequence of individual en-route travel decisions. For more details about the traffic and public transport supply representation see Toledo et al. (2010). The dynamic public transport path choice model and related congestion effects is described in Cats et al. (2016) and the modelling of unplanned service disruptions and their impacts on passenger distribution are explained in Cats and Jenelius (2015). The reader is referred to these studies for further details while the essential modelling principles are briefly provided below.

The different sources of public transport operations uncertainty are modelled explicitly, including traffic conditions, vehicle capacities, dwell times, vehicle schedules and service disruptions. BusMezzo is an event-based simulator which models transit vehicles individually, but does not represent explicitly the lanes of the infrastructure. Links in BusMezzo are divided into two parts: a running part and a queuing part. Travel times on the running part are determined by a speeddensity function. At the downstream part of the link, the vehicles join a single queue of vehicles waiting to move out of the link. Queue servers process the vehicles in this queue and into the successive link until it is not full. Separate queue servers with their corresponding capacities are used for each turning movement in order to capture link connectivity and lane channelling. A headway-based control is applied to rail-bound services to mimic traffic signal control. Public transport vehicles are characterised by length, number of seats, capacity, control regime. Vehicles follow a list of 
scheduled trips. Because of this, it is possible to model explicitly trip chaining in the trip sequence. A public transport line is defined by its origin and destination terminals and by the sequence of stops that it serves in between. Each line is served by a specified number of trips, determined by the number of vehicles that depart. In the context of high-frequency metropolitan services, passengers do not consult the timetable to coordinate their arrival with the scheduled vehicle arrival time but rather arrive at random at stops. Passenger arrival at stops is described as a random Poisson arrival process with average arrival rates specified in time-dependent OD matrices. A dynamic path choice model considers each traveller as an adaptive decision maker who progress in the transit system by means of successive decisions. A non-equilibrium network loading is performed with probabilistic path choice (Cats et al. 2011). This analysis allows identifying the set of network elements to which networks are most vulnerable in the event of a disruption, resulting with severe consequences.

As an event-based simulator, the time clock of the simulation progresses from one event to the next according to a chronological list of events that refers to the relevant objects. During the simulation, for each vehicle, updated passengers loads are recorded and determine crowding levels and the maximum number of passengers that may board at each stop. Outputs generated by the model include time-dependents measures of performance at the stop, trip and line level (dwell times, early and late arrivals, travel times between stops), passengers travel time distributions and bus loads.

\subsection{Measuring the impacts of service disruptions}

To measure the change generated in a system by a disruption, a general approach is to describe the new and the baseline state, and assess the difference in system performance measures. Such indicators should provide information on locations in the transport system where disruptions cause the most adverse conditions and therefore should be prioritized for the implementation of prevention and mitigation. In this study, our aim is to identify the impacts that the closure of a service segment may cause by examining network passengers flows and flow redistribution effects. The analysis of passenger flows can detect the most overloaded links in the network and shows if the service supply is able to absorb the diverting demand.

The analysis entails two phases: identification and evaluation. In the former, the most "central" links in the network are selected for detailed investigation. Central links are those which give rise to the worst consequences in case they fail. In this study we focus on travellers' perspective and therefore measure centrality using the Passenger Betweenness Centrality (PBC) indicator proposed in Cats and Jenelius (2014). A link $e$ belonging to a certain path $j$ may thus be considered central if it is used by a large number of passengers. With $\delta(j, e)$ being equal to 1 if $e \in j$ and 0 otherwise, the link betweenness centrality measure from the travellers' perspective is:

$$
P B C(e \mid t, \tau)=\frac{\sum_{j \in J} \mathbb{E}\left[\sum_{n \in N(t, \tau)} p_{n}(j)\right] \delta(j, e)}{\mathbb{E}[N(t, \tau)]}
$$

Where $N(t, \tau)$ is the set of travellers between all OD pairs during the time interval $(t, t+\tau) ; p_{n}(j)$ is the probability that passenger $n$ chooses the path $j ; J$ is the set of all paths. It is possible to note that the numerator is simply the expected total number of travellers traversing link during the time interval, while the denominator is the expected number of travellers in the system during the same period.

In the second phase, a disruption scenario is simulated considering the closure of one candidate central links. It is then necessary to define a performance indicator that will reflect the impact of link failure. The vulnerability of the network is assessed by the difference between the values of this indicator in the disrupted and the base (i.e. normal operations) case.

In this study, the link-specific performance indicator chosen is the Volume Over Capacity ratio (VOC). VOC is measured at individual trip level. For vehicle $m$ traversing link $e$ at time $t$ in the scenario $s$ it is given by:

$$
\operatorname{VOC}_{m}(e, t \mid s)=\frac{q_{m}(e, t)}{\kappa_{m}}
$$

Where $q_{m}(e, t)$ is the number of passengers on board vehicle $m$ on link $e$ at during time window $t$; $\kappa_{m}$ is the capacity of vehicle $m$. This indicator measures link on-board saturation level. High saturation levels cause on-board overcrowding. When it equal to the upper limit of 1 , some passengers may experience denied boarding and thus prolonged waiting times. Unlike static assignment models in which volumes can (unrealistically) exceed capacity $(V O C>1)$ implying that the network cannot absorb the loaded demand, the dynamic assignment model used in this study enforces strict capacity constraints at individual vehicle level and hence ensures $0 \leq$ VOC $\leq 1$ (Cats and Hartl 2016).

The impact of the closure of a certain link on the network is computed comparing this indicator in the base scenario $s_{b}$ and in the disrupted scenario $s_{d}$. The comparison is made using two different network-wide indicators that average the 
absolute and relative difference of saturation level over all transit vehicles, namely the Actual Difference Ratio (ADR):

$$
A D R\left(t \mid s_{d}\right)=\frac{\sum_{e \in E} \sum_{m \in M_{t, e, s}}\left(V O C_{m}\left(e, t \mid s_{d}\right)-V O C_{m}\left(e, t \mid s_{b}\right)\right)}{\sum_{e \in E}\left|M_{t, e, s_{d}}\right|}
$$

and the Relative Difference Ratio (RDR):

$$
R D R\left(t \mid s_{d}\right)=\frac{\sum_{e \in E} \sum_{m \in M_{t, e, s_{d}}}\left(\frac{V O c_{m}\left(e, t \mid s_{d}\right)-V O c_{m}\left(e, t \mid s_{b}\right)}{V O c_{m}\left(e, t \mid s_{b}\right)}\right)}{\sum_{e \in E}\left|M_{t, e, s_{d}}\right|}
$$

Where $M_{t, e, s_{d}}$ is the set of vehicles traversing link $e$ during time window $t$ in the scenario $s_{d}$.

Since both ADR and RDR refer to average changes in link saturation levels, they are expected to be different from zero when the network is disrupted due to the redistribution of passenger flows. Critical links are defined as those whose disruption results in the greatest impacts, i.e. the highest values of ADR and RDR. ADR can be used to estimate the recovery time, i.e. the time the system needs to return to its initial performance level. When the ADR approaches zero, the network has returned to the baseline condition and the impacts provoked by the disruption has been adsorbed. The impacts of the disruption are likely to increase with the recovery time in the absence of disruption management measures.

\section{Application}

\subsection{Network description}

We illustrate our approach by an application to the Stockholm rapid transit system (Figure 1), made up of seven Metro lines (lines 10, 11, 13, 14, 17, 18, 19), four high-demand trunk bus lines (lines 1, 2, 3, 4) and one light rail train line (line 22). The Stockholm network consists of a mature and dense multi-modal core that offers route choice alternatives and the system is subject to recurrent disruptions, especially under adverse weather conditions.

The three transit modes are modeled with different vehicle types, operating speeds, travel time variability, dwell time functions and holding strategies to reflect the differences in their service characteristics. Key attributes of each public transport mode and the corresponding lines are shown in Table 1.

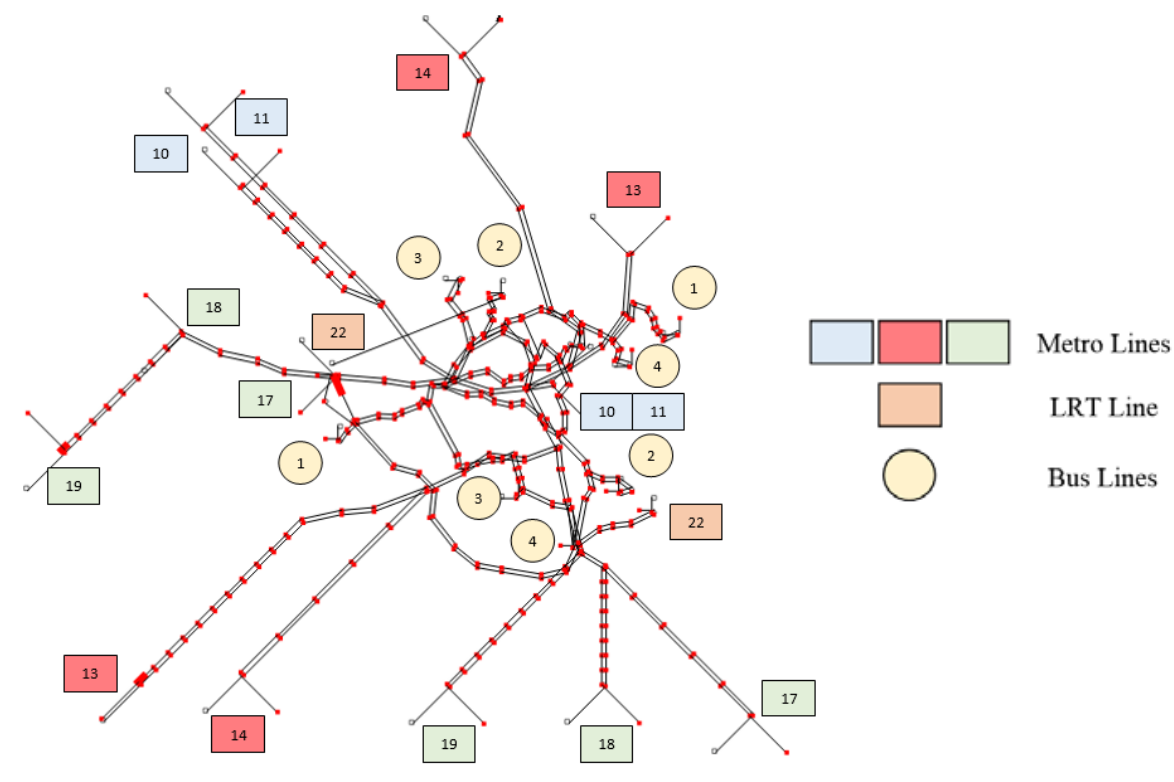

Figure 1: Stockholm rapit transit network as displayed by BusMezzo

Table 1: Service mode attributes

\begin{tabular}{ccccc}
\hline MODE & LINES & SEATS & CAPACITY & SCHEDULED HEADWAY [min] \\
\hline METRO & $10,11,13,14,17,18,19$ & 300 & 1000 & 5
\end{tabular}




\begin{tabular}{lcccc} 
LRT & 22 & 100 & 200 & 7.5 \\
BUS & $1,2,3,4$ & 55 & 110 & 5 \\
\hline
\end{tabular}

The network, including 437 stops, is coded in BusMezzo using the estimated real-world demand, public timetables and estimated walking distances between platforms and stops. Approximately 800 vehicle trips take place in the morning peak-period (6:00-9:00), operated by 204 vehicles. System performance is evaluated for the period between 7 and $8 \mathrm{am}$. In the simulation, more than 123,000 passenger journeys start during this hour.

The parameters of the choice generation model and the dynamic path choice model were estimated by a statedpreference survey on public transport route choice decision (Cats et al., 2011). The coefficients of the utility function were found to be $\beta_{a}^{\text {wait }}=-0.07, \beta_{a}^{\text {walk }}=-0.07, \beta_{a}^{\text {ivt }}=-0.04, \beta_{a}^{\text {trans }}=-0.334$. This reflects a ratio of 1.75 between in-vehicle and waiting or walking times and a transfer penalty equivalent approximately to eight in-vehicle minutes. Travelers' preferences are considered constant throughout the assignment period. Trip fare is zone-based and thus fixed in the Stockholm network for a given OD pair and passenger group and therefore does not affect passenger path decisions.

Real-time Information (RTI) is assumed to be available to passengers at origins, at stops and rail stations. Passenger receive information on the expected remaining time to the next vehicle arrival for each service line, from all stops within direct proximity (i.e. public transport hub). It is assumed that passengers utilize real-time information whenever available to adjust their pre-trip access stop choice or reroute based on real-time forecasts at stops. In other words, information is available to passengers only at passenger path $j$ nodes, i.e., passengers that depart after the disruption has commenced as well as passengers waiting at stops upstream or downstream of the disrupted line-segment. In contrast, onboard passengers do not have information on downstream disruptions.

The base scenario is used to compute PBC (see Eq. 2) across the network. We analyze the impact in terms of VOC of a disruption occurring on the five network corridors with the highest PBC (Table 2). All the chosen segments are in the core of the network, where there are rapid public transport alternatives. They are all metro segments.

\begin{tabular}{cccc} 
Table 2: Disruption scenarios & & \\
\hline DISRUPTION CASE & DISRUPTED LINE & DISRUPTED SEGMENT & $\begin{array}{c}P B C(e \mid t, \tau) * E[|N(t, \tau)|] \\
\text { Average passenger load in the } \\
\text { peak hour }\end{array}$ \\
\hline D1 & $17,18,19$ (Northbound) & Gullmarsplan - Hotorget & 27358 \\
D2 & 13,14 (Northbound) & Liljeholmen - Centralen & 17432 \\
D3 & $17,18,19$ (Southbound) & Alvik - Centralen & 10944 \\
D4 & 10,11 (Southbound) & Fridhemsplan - Centralen & 10183 \\
D5 & 13,14 (Southbound) & Centralen - Hornstull & 8983 \\
\hline
\end{tabular}

In each scenario, we simulate an unplanned closure of the disrupted segment between 7:15 and 7:45. Since the disruption is short and unplanned, the service operator cannot apply mitigation measures, such as providing a replacement service or operating the remaining disconnected parts of the line as independent lines. Note that the closure of a segment of a certain line does not prevent other lines from running because the metro lines in the case study network have separate infrastructure - tracks and platforms - as well as fleet. The simulation model prevents vehicles from traversing the disrupted link as long as the disruption is in effect. This implies that upstream public transport vehicles progress until they queue upstream of the disruption. On-board passengers are unable to alight while passengers waiting at downstream stops (including stops along the disrupted element) can reconsider and revise their travel decisions. Figure 2 shows the disrupted segment in each of the five scenarios. 

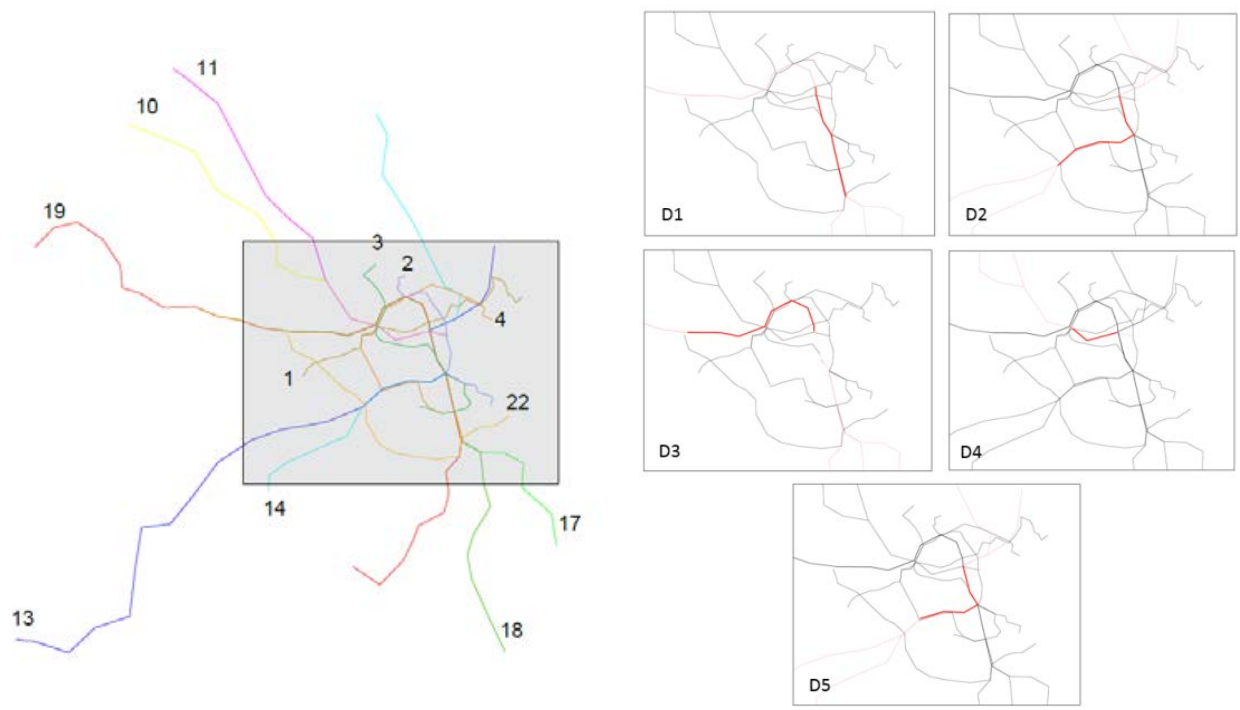

Figure 2: Maps of the disruptions

For each scenario, we carry out 10 simulation runs for a three hours' period with a uniform passenger demand during the peak hour. This number of runs leads to a maximum error of less than $5 \%$ for the passenger travel time.

\subsection{Results}

\subsubsection{Temporal propagation}

Under normal operational conditions, the average passenger travel time in the case study network is 25 minutes. When the flows in the network reach their maximum value (approximately during 7:30-8:30AM, Figure 3a), the majority of the links have a VOC between 0.1 and 0.3 and only few services (1\%) cannot satisfy all the demand because they reach capacity (see Figure 3b). In $29 \%$ of all service segments, VOC exceeds 0.3, implying that there are standees as the passenger volume surpasses the number of seats (i.e. load factor larger than 1). A large share of the network lines is located on line branches which typically carry low passenger volumes. Moreover, the results report the distribution over links and not over passengers which on average experience more congested conditions. In the disrupted scenarios, the number of congested links increases but very slightly because oversaturation effects and these effects are mostly confined to the core of the network.

a)

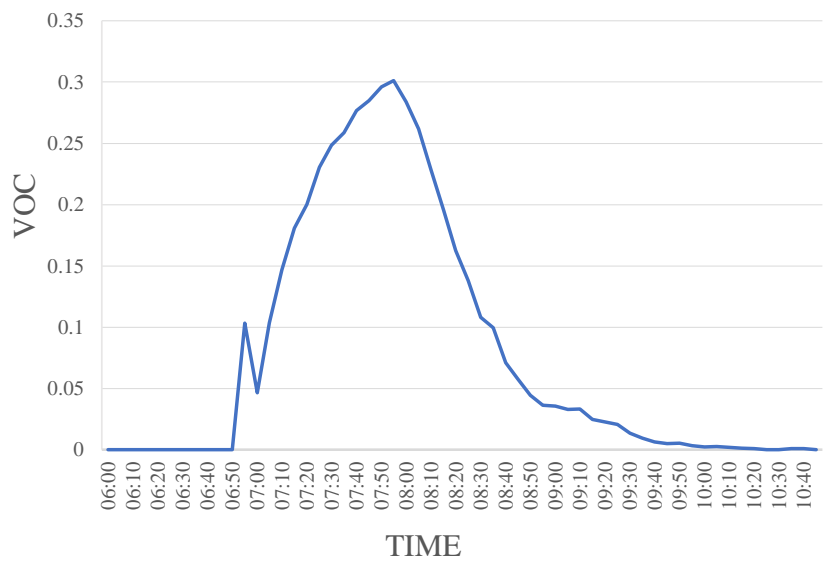

b)

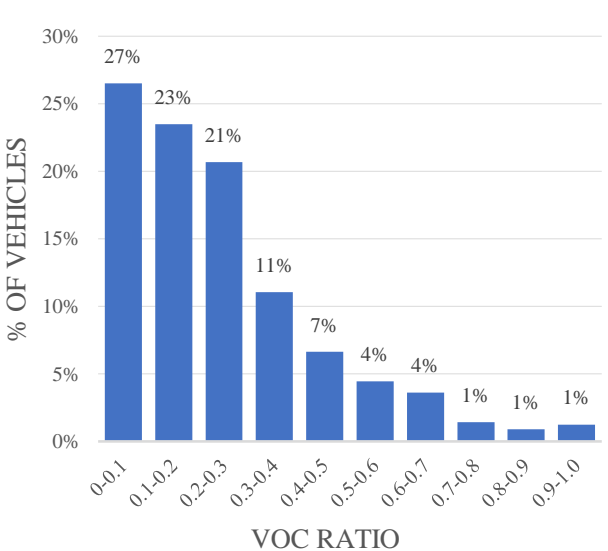

Figure 3: a) Occupancy in the base scenario. Data are averaged over time slices of 5 minutes;

b) Percentage of vehicles with a given VOC ratio in the time interval 7.30 - 8.30 AM

Figure 4 shows, for each scenario, the temporal profile of $R D R$. Data is averaged over time slices of 5 minutes. Time is computed from the beginning of the simulation (i.e. zero corresponds to $6.00 \mathrm{AM}$ ). The qualitative trend of these 
indicators is almost the same in all scenarios, even though the magnitude of RDR vary significantly with the scenario. When the generation of passengers starts (1 hour into the simulation) there is a sharp increase in RDR - in one case an increase of more than $600 \%$ - due to passengers who intended to use the disrupted link and are forced to change their path. This sharp and abrupt increase in the case of D1 of D5 occurs because of the redistribution of passengers from disrupted metro links to alternative underutilized bus service segments that then result in a large relative increase in passenger volume from an initially low level. After that, the indicator stabilizes at a level of 0.2-0.3, implying an increase of $20-30 \%$ increase in the average VOC per link with consequences on passenger travel comfort.

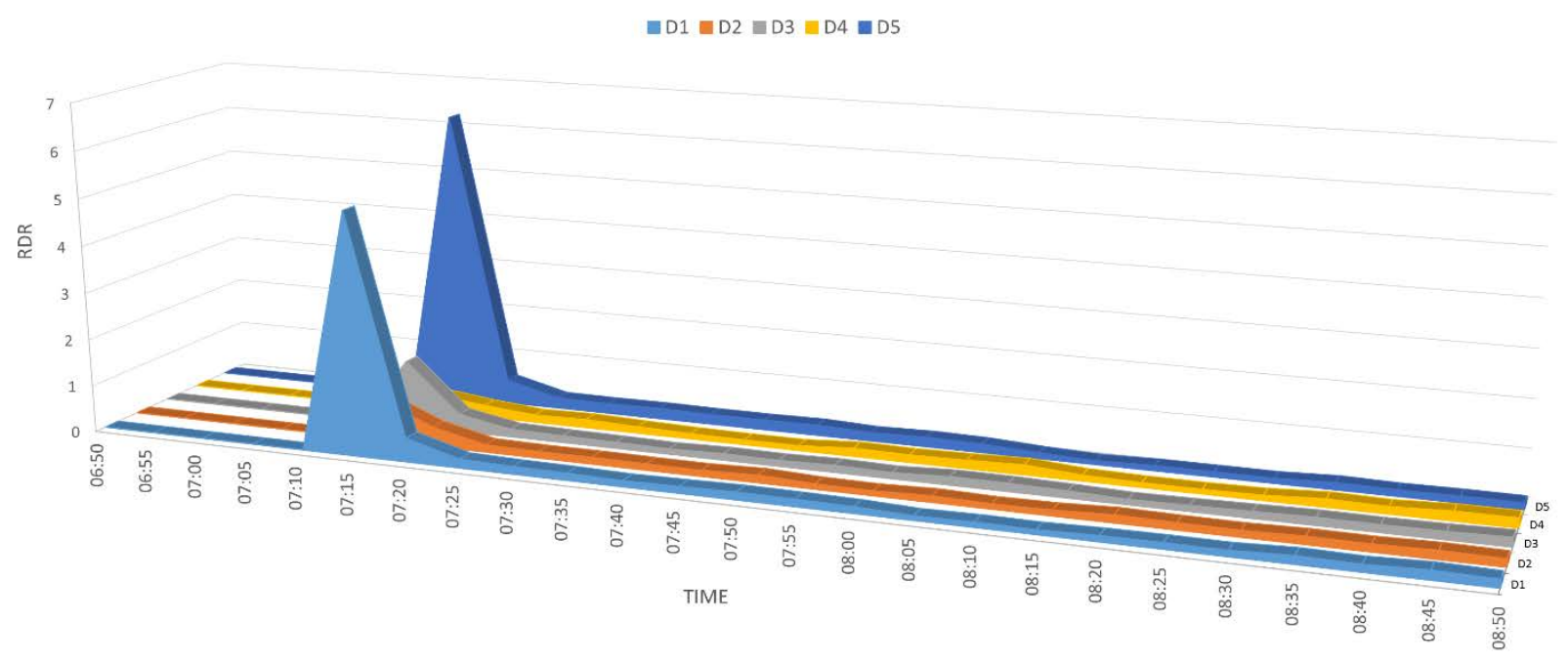

Figure 4: Relative difference ratio in volume over capacity evolution for each disruption scenario.

Figure 5 shows the temporal profile of the ADR indicator. As in the case of RDR, the overall trend follows a similar pattern for all disruption scenarios. It is evident that the ADR indicator increases sharply following the disruption start at 7:15 AM. The absolute change in VOC is most severe in the case of D4 which disrupts a central high-volume segment, resulting in a large increase in passenger volumes and hence VOC on parallel services. It then falls into a medium level that persists for approximately an hour after the disruption ended, implying an average increase of 0.02-0.05 in the VOC value per link. However, the spatial distribution of this change is remarkably uneven as discussed below in section 3.2.3. Afterward, the difference between the base case and the disrupted cases progressively diminishes.

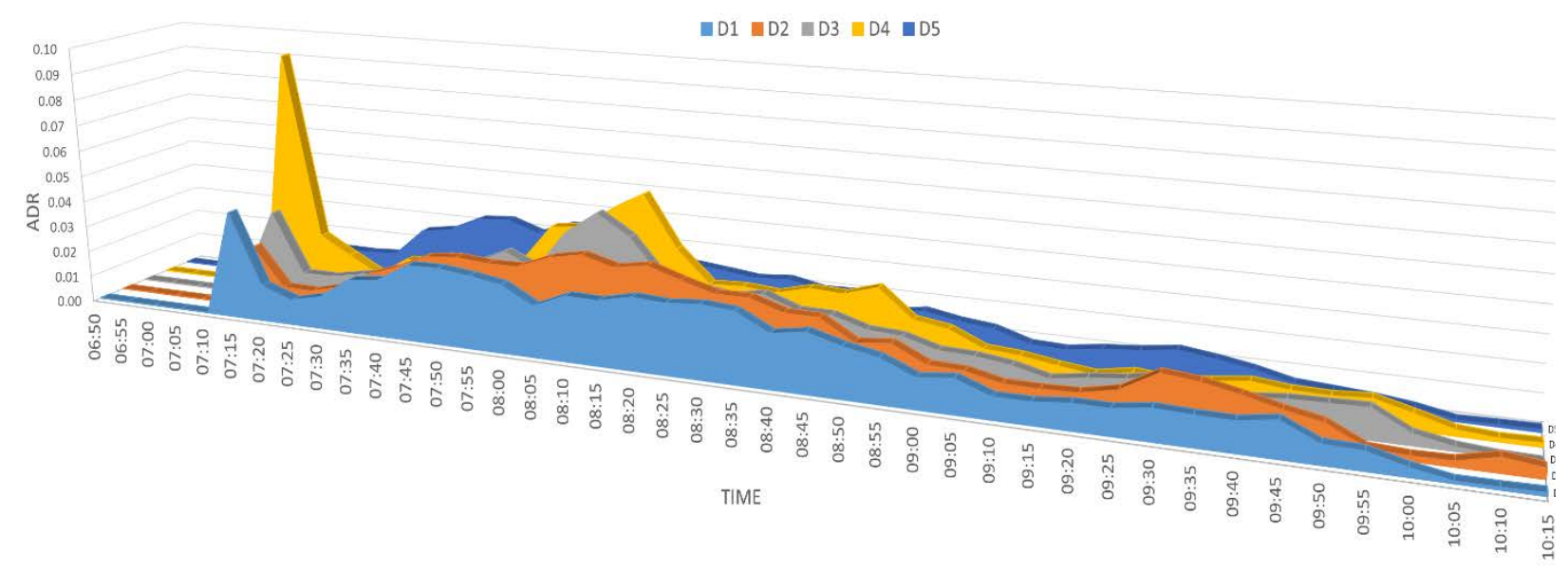

Figure 5: Absolute difference ratio in volume over capacity evolution for each disruption scenario.

While the previous graphs allow identifying the scenarios with the highest initial impacts, in order to measure the vulnerability of the network over the entire study period, we need an aggregate indicator, which we will call Networkwide Link Saturation. For each scenario, the area under the curve in Figure 5 is an indicator of the total increase in VOC across the network: 


$$
N L S=\int A D R d t
$$

The larger the value of $N L S$, the more adverse the impacts of the disruption are in terms of network-wide increase in link saturation. Table 3 shows the value of this indicator for each scenario.

\begin{tabular}{|c|c|c|c|c|c|}
\hline SCENARIO & D1 & D2 & D3 & D4 & D5 \\
\hline NLS & 100.03 & 113.38 & 99.06 & 112.77 & 90.33 \\
\hline
\end{tabular}

The scenario in which the network is most disrupted is D2, closely followed by D4, while the case with least impact is D5. While D4 induces more acute effects on network saturation, D2 is characterized by a more chronic increase in volume over capacity levels. The results suggest that there is a loose relation between the ranking attained by centrality measures (Eq. 2) which was used in the enumeration of D1-D5, and the importance of the link to network vulnerability as measured in this study (Eq. 6).

\subsubsection{Recovery time}

The network recovery time - the time required for the network to return to undisrupted conditions (base scenario level), is computed as the time at which $\mathrm{ADR} \rightarrow 0$ (Figure 5). For all scenarios, the recovery time is between 4 and 4.5 hours after the beginning of the simulation, which corresponds to 10:00-10:30 AM, or 2.5 hours after the actual disruption ended. These results suggest that the aftermath of a disruptions last up to 5 times longer than the disruption itself. The scenario with the shortest recovery time is D3, while D2 has the longest recovery time. Simulations with demand generated for a longer time (between 7.00 and 9.00 in the morning) show the same recovery time and trends. This confirms that the system recovers not because there are no more passengers in the simulation, but rather because the network effectively returns to its original state.

Table 4: Recovery time for each disrupted scenario, computed from the end of the disruption (7.45 AM)

\begin{tabular}{cccccc}
\hline SCENARIO & D1 & D2 & D3 & D4 & D5 \\
\hline Recovery time (hours) & 2.47 & 3.05 & 2.80 & 3.05 & 2.97 \\
\hline
\end{tabular}

In order to examine how the temporal extent of the perturbation influences the recovery time, simulations were run changing the duration of the disruption. Recalling that in the previous analysis the closure lasted 30 minutes (between 7.15 and $7.45 \mathrm{AM}$ ), additional simulation scenarios were devised with shorter (between 7.15 and 7.30 AM, 15 minutes) and longer disruptions (between 7.15 and 8.00 AM, 45 minutes). In both cases, the trend of the RDR and ADR remained the same as in the original case, with a steep increase at the beginning and, followed by more modest values and a similar recovery time.

\subsubsection{Spatial propagation: Spillover effects}

The spatial propagation of spillover effects is analysed for each scenario to quantify the network-wide effects provoked by each disruption. Figure 6 shows, for each scenario, the distance at which the maximum value of ADR was obtained (corresponding to the radius of the blue circumferences) and displays the line with the largest increase in volume over capacity. The latter is often one of the lines that traverses the disrupted segment because of the knock-down effects in the post-disruption pre-recovery period for those passengers in the periphery of the network that have no possibility to reroute. Table 5 reports the distance at which the ADR reaches its maximum value, the corresponding ADR peak value, the 30minutes time window during which it is observed and the most affected public transport line for each scenario. The impacts of disruption scenarios with low NLS values are not necessarily confined to smaller areas. For example, D5 has the lowest NLS value but its influence extends to links located in the outskirts and the influence area encompasses the entire network, similarly to D2 which has the highest NLS value. If the most adversely affected links are characterized by low VOC ratios in the base case, then even if the impacts are higher, there is a sufficient residual capacity to accommodate the rerouting passengers. 


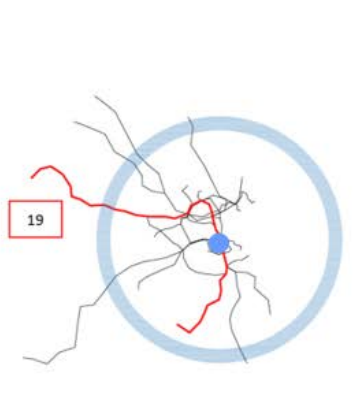

D1

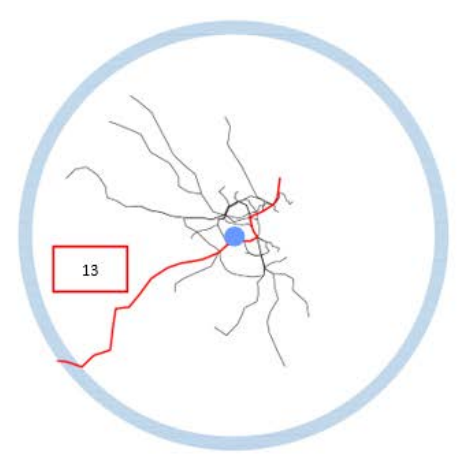

D2

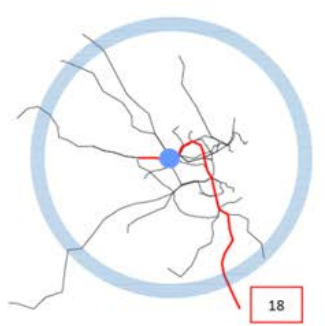

D3

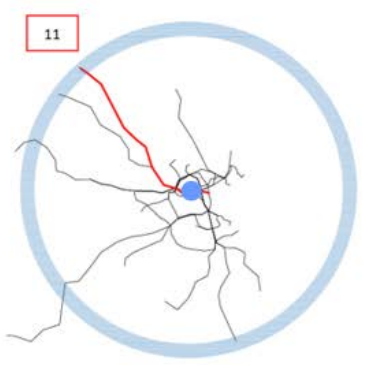

D4

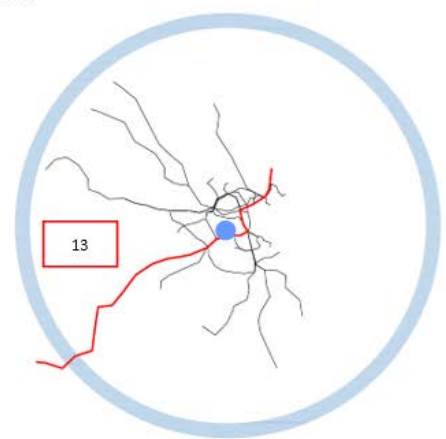

D5

Figure 6: Extent and location of the maximum impact in each scenario.

Table 5: Location and time of the maximum impact in each scenario

\begin{tabular}{ccccc}
\hline SCENARIO & ADR & LINE & DISTANCE (m) & TIME \\
\hline D1 & 0.08 & 19 & 8500 & $7.30-8.00 \mathrm{AM}$ \\
D2 & 0.08 & 13 & 15000 & $8.00-8.30 \mathrm{AM}$ \\
D3 & 0.14 & 18 & 9500 & $7.30-8.00 \mathrm{AM}$ \\
D4 & 0.13 & 11 & 11500 & $7.30-8.00 \mathrm{AM}$ \\
D5 & 0.22 & 13 & 14000 & $7.30-8.00 \mathrm{AM}$ \\
\hline
\end{tabular}

\section{Conclusions}

The attract more passengers. public transport system must be able to withstand or recover quickly from disruptions such as infrastructural and vehicular malfunctions. Because of the dynamic nature of the public transport system, disturbances propagate across the network due to knock-down effects on the infrastructure and spillover effects caused by the redistribution of passenger flows and capacity limitations. To study these effects, we utilize a demand loading simulation model that takes into consideration the interaction between supply and demand and the inherent stochastic processes in the public transport system. The impacts of disruptions are analysed studying the spatial and temporal change of the Volume over Capacity ratio over the network. We define two indicators that provide information on the network saturation level and help understanding if, after a disruption, to what extent the transit supply can satisfy the demand.

The proposed method was applied to a series of selected disruption scenarios of unplanned breakdowns of the most central service corridors for the rapid metropolitan network of Stockholm. The application demonstrates that the impact of disruptions on passenger flow distribution last up to 6 times longer than the service closure time. Furthermore, significant impacts of the disruption are observed more than 10-15 km from the disruption location. Our findings also show that the centrality measure is not indicative of the scenarios with higher impacts on network performance.

The disruption propagation analysis of public transport networks will facilitate the development of measures for improving system performance in planning, operations and real-time management. It allows identifying adverse scenarios, expressed as a set of important network elements whose disruptions would cause the worst consequences. The identification of such links can provide guidelines for infrastructure and service investment decisions. Timetable and vehicle scheduling design considering risk distribution may reduce the likelihood of disruption, while better management 
and restoration strategies will reduce or encapsulate its impact.

Future studies may consider other types of failures such as node closures. The consequences of the simultaneous failure of multiple links should be analysed. The methodology should be expanded to include the probability of a link to be disrupted. The relationship between the results obtained from this study and some topological network properties such as the connectivity should also be investigated. Finally, an empirical network analysis of the recovery times and spillover effects of service disruptions would validate the approach.

\section{References}

Angeloudis, P., Fisk, D., 2006. Large subway systems as complex networks. Physica A: Statistical Mechanics and its Applications, 367, 553-558.

Ash, J., Newth, D., 2007. Optimizing complex networks for resilience against cascading failure. Physica A 380, 673-683.

Barker, K., Ramirez-Marquez, J.E., and Rocco, C.M., 2013. Resilience-based network component importance measures. Reliability Engineering \& System Safety 117, pp. 89-97.

Berche, B., von Ferber, C., Holovatch, T., Holovatch, Y., 2009. Resilience of public transport networks against attacks. Eur. Phys. J. B 71, 125-137.

Cats O., Hartl M., 2016. Modelling public transport on-board congestion: Comparing schedule-based and agent-based assignment approaches and their implications. Journal of Advanced Transportation, 50 (6), 1209-1224.

Cats, O., Jenelius, E., 2015. Planning for the unexpected: the value of reserve capacity for public transport network robustness. Transportation Research Part A: Policy and Practice 81, 47-61.

Cats, O., Jenelius, E., 2014. Dynamic vulnerability analysis of public transport networks: mitigation effects of real-time information. Networks and Spatial Economics 14 (3-4), 435-463.

Cats, O., Jenelius, E., 2016. Beyond a complete failure: the impact of partial capacity degradation on public transport network vulnerability. Tranportmetrica B: Transport Dynamics. doi.org/10.1080/21680566.2016.1267596.

Cats, O., Koutsopoulos, H.N., Burghout, W., Toledo, T., 2011. Effect of real-time transit information on dynamic passenger path choice. Transportation Research Record 2217, 46-54.

Cats O., West J., Eliasson J., 2016. A dynamic stochastic model for evaluating congestion and crowding effects in transit systems. Transportation Research Part B: Methodology 89, 43-57.

De-Los-Santos, A., Laporte, G., Mesa, J. A., Perea, F., 2012. Evaluating passenger robustness in a rail transit network. Transportation Research Part C: Emerging Technologies, 20 (1), 34-46.

Derrible, K., Kennedy C., 2010. The complexity and robustness of metro networks. Physica A 389 (17), 3678-3691.

Han, C., Liu, L., 2009. Topological vulnerability of subway networks in China. International Conference on Management Service Science in Wuhan 20-22 Sept. 2009, IEEE.

Hollnagel, E., 2011. Prologue: the scope of resilience engineering. In: Hollnagel, E., Dédale, J.P., Woods, D., Wreathall, J. (Eds.), Resilience Engineering in Practice: A Guidebook. Ashgate, pp. xix-xxxix.

Holme, P., Kim, B. J., Yoon, C. N., Han, S. K., 2002. Attack vulnerability of complex networks. Physical review E, 65 (5), art. no. 056109.

Latora, V., Marchiori, M., 2005. Vulnerability and protection of infrastructure networks. Physical Review E, 71 (1), art. no. 015103.

Rodríguez-Núñez, E., García-Palomares, J. C., 2014. Measuring the vulnerability of public transport networks. Journal of transport geography, 35, 50-63.

Shelat, S., Cats, O., 2017. Measuring spill-over effects of disruptions in public transport networks. The 5th IEEE International Conference on Models and Technologies for Intelligent Transportation Systems (MT-ITS2017), Naples, 756-761.

Taleb, N.N., 2014. Antifragile: Things That Gain From Disorder. Random House, New York.

Toledo, T., Cats, O., Burghout, W., Koutsopoulos, H.N., 2010. Mesoscopic simulation for transit operations. Transportation Research Part C: Emerging Technologies 18 (6), 896-908.

Von Ferber, C., Berche, B., Holovatch, T., Holovatch, Y., 2012. A tale of two cities. Journal of Transportation Security, 5 (3), $199-$ 216.

Von Ferber, C., Holovatch, T., Holovatch, Y., Palchykov, V., 2009. Public transport networks: empirical analysis and modeling. The European Physical Journal B-Condensed Matter and Complex Systems, 68 (2), 261-275.

Zhang, J., Xu, X., Hong, L., Wang, S., Fei, Q., 2011. Networked analysis of the Shanghai subway network, in China. Physica A 390, 4562-4570. 